INPLASY

PROTOCOL

To cite: Chen et al. Fire needle for recurrent aphthous stomatitis A protocol for systematic review and network meta-analysis. Inplasy protocol 2021120118. doi:

10.37766/inplasy2021.12.0118

Received: 26 December 2021

Published: 26 December 2021

Corresponding author: Jun Chen

juston521@163.com

Author Affiliation:

College of Acupuncture and Massage, Shandong University of Traditional Chinese Medicine, JinanShandong University of Traditional Chinese Medicine.

Support: SDYAL21058.

Review Stage at time of this submission: Data extraction Completed but not published.

\section{Fire needle for recurrent aphthous stomatitis A protocol for systematic review and network meta-analysis}

Chen, J1; Wang, XX2; Wang, K³; Liu, ZH4; Lv, XX5; Wang, M6; Sun, SD?.

Review question / Objective: Results: This study will provide a comprehensive review of current evidence for the treatment of fire needle on RAS. Conclusion: The conclusion of this study will provide a judging basis that whether the treatment of RAS with fire needle is effective. PICOS will be applied, including Population, Intervention,Comparison, Outcome, and Study.

Information sources: English databases(PubMed, Embase, and Web of Science), Chinese databases(CNKI, Wanfang database, CBM, and VIP) and clinical registration platforms (Cochrane Library, Chinese Cochrane Centre's Clinical Trial RegistryPlatform).

INPLASY registration number: This protocol was registered with the International Platform of Registered Systematic Review and Meta-Analysis Protocols (INPLASY) on 26 December 2021 and was last updated on 26 December 2021 (registration number INPLASY2021120118).

Conflicts of interest:

None declared.

\section{INTRODUCTION}

Review question / Objective: Results: This study will provide a comprehensive review of current evidence for the treatment of fire needle on RAS. Conclusion: The conclusion of this study will provide a judging basis that whether the treatment of RAS with fire needle is effective. PICOS will be applied, including Population, Intervention, Comparison, Outcome, and Study.

Condition being studied: Recurrent aphthous stomatitis (RAS), are common inflammatory lesions of the oral mucous, usually round or ovoid, circumscribed by 
erythematous haloes with a yellow-grey floor and mostly painful. RAS patients usually experience prodromal burning sensations that last from 2 to 48 hours before an ulcer appears. Ulcers are round with well-defined erythematous margins and a shallow ulcerated center covered with yellowish-gray fibrinous pseudomembrane. RAS ulcers usually develop on non-keratinized oral mucosa, with the buccal and labial mucosa being the most common sites, and last approximately 10 to 14 days without scar formation. RAS is often idiopathic but can be associated with gastro-intestinal diseases (i.e. celiac disease, inflammatory bowel diseases), nutritional deficiencies (iron, folates...), immune disorders (HIV infection, neutropenia) and rare syndromes. Approximately $20 \%$ of the general population is affected by RAS, but incidence varies from $5 \%$ to $50 \%$ depending on the ethnic and socioeconomic groups studied 2,3 . The prevalence of RAS is influenced by the population studied, diagnostic criteria, and environmental factors 1. In children, prevalence of RAS may be as high as $39 \%$ and is influenced by the presence of RAS in one or both parents 4 . Children with RASpositive parents have a $90 \%$ chance of developing RAS compared with $20 \%$ of those with RAS-negative parents 2. In children of high socioeconomic status, RAS is five times more prevalent and represents $50 \%$ of oral mucosal lesions in this cohort 5, 6. RAS prevalence was found to be higher (male, $48.3 \%$; female, $57.2 \%$ ) among professional school students than in the same subjects 12 years later when they had become practicing professionals. This finding led some investigators to theorize that stress during student life is a major factor in RAS, although the differences due to age changes should also be considered. The onset of RAS appears to peak between the ages of 10 and 19 years and becomes less frequent with advancing age, geographic location or gender7. If RAS begins or significantly increases in severity after the third decade and well into adult life (see Table 1), it should increase suspicion that the etiology of the condition maybe attributed to an underlying medical disorder such as hematologic, immunologic, connective tissue disease, or Behçet's syndrome. Many treatments have been advocated for recurrent aphthous ulceration. These may be based upon antiseptics, antibiotics, corticosteroids, immunosuppressants, antirheumatics, anti-inflammatories, hormone therapy, antivirals, colchicine, thalidomide, pentoxifylline, sodium cromoglycate, interferon, hyaluronic acid, helicobacter eradication, zinc, various acids, gastric ulcer treatments, ultrasound, laser, cautery, cryotherapy, bioadhesives, herbal remedies, homeopathy, vitamins, lactobacillus as well as sundry other management strategies and combinations of various medications.4, 5, 31 Systemic treatment may be appropriate for more severe and resistant cases. It should be made clear to the patient that the objective of treatment is symptomatic and that the ulcers cannot be "cured". The plethora of treatments used for the treatment of oral ulceration is testament to the lack of any single effective treatment. There has not been a systemic (Cochrane) review of oral ulceration published. In China, acupuncture and moxibustion are effective traditional therapeutics and fire needle is an operation method in traditional acupuncture therapy. Fire needle with rapid needle, through the sudden warming point to the body to pass nerve, body temperature stimulation information reaction, so as to strengthen the body ' $s$ immune response to the disease, promote lesion repair.As an nondrug therapy, fire needle has been reported in some clinical studies that has certain curative effect on RAS. Therefore, the purpose of this study was to summarize the original research on the treatment of RAS with fire needle, so as to evaluate whether the treatment of RAS with fire needle is really effective.

\section{METHODS}

Search strategy: The two authors will independently search English databases (PubMed, Embase, and Web of Science), Chinese databases(CNKI, Wanfang database, CBM, and VIP) and clinical registration platforms (Cochrane Library, 
Chinese Cochrane Centre's Clinical Trial Registry Platform). The search time is from the establishment of the database to December, 2021. Boolean algorithm is used as search formula to search full-text articles with subject terms and free words. Search: $1(1(1(1)(()($ recurrent aphthous stomatitis[Title/Abstract]) OR (Stomatitis, Aphthous[Title/Abstract])) OR (Aphthous Stomatitides [Title/Abstract])) OR (Aphthous Stomatitis[Title/Abstract])) OR (Stomatitides, Aphthous[Title/Abstract])) OR (Ulcer, Aphthous [Title/Abstract])) OR (Aphthae[Title/Abstract])) OR (Canker Sore[Title/Abstract])) OR (Canker Sores[Title/Abstract])) OR (Sore, Canker[Title/Abstract])) OR (Sores, Canker[Title/Abstract])) OR (Periadenitis Mucosa Necrotica Recurrens[Title/ Abstract])) AND (((()fire needle[Title/ Abstract]) OR (Cautery[Title/Abstract])) OR (huo zhen[Title/Abstract])) OR (fire needle moxibustion [Title/Abstract])) OR (acupuncture[Title/Abstract]))) AND (((andomized controlled trial[Title/Abstract]) OR (randomized[Title/Abstract])) OR (placebo[Title/Abstract])).

Participant or population: Participants diagnosed as PCs by clinicians referring to the New Routine for Diagnosis and Treatment will be included. No restrictions on gender, age, race, etc.

Intervention: Types of interventions. Without limits on course and dose, we will include studies in which fire needle is the primary intervention and, if necessary, we will include studies in which fire needle is combined with other active treatments versus active treatment alone.

Comparator: Types of comparisons.The selected RCTs should testify that the interventions were compared with a control group composed of placebo, sham acupuncture, no treatment, or other active therapies.

Study designs to be included: Types of studies.Randomized controlled trials (RCTs) with fire needle as the primary intervention for RAS will be included, and other studies such as case reports, and reviews will be excluded. No restrictions on country but language will be limited on English and Chinese.

Eligibility criteria: This systematic review will aim to evaluate the effect and safety of fire needle therapy for PCs. Our protocol has been registered on the International Platform of Registered Systematic Review and Meta-Analysis Protocols (INPLASY).All steps of this systematic review will be performed according to the Cochrane Handbook. 2.2.1. Types of studies. Randomized controlled trials (RCTs) with fire needle as the primary intervention for RAS will be included, and other studies such as case reports, and reviews will be excluded. No restrictions on country but language will be limited on English and Chinese. 2.2.2. Types of participants. Participants diagnosed as PCs by clinicians referring to the New Routine for Diagnosis and Treatment will be included. No restrictions on gender, age, race, etc.2.2.3. Types of interventions. Without limits on course and dose, we will include studies in which fire needle is the primary

Information sources: English databases (PubMed, Embase, and Web of Science), Chinese databases (CNKI, Wanfang database, CBM, and VIP) and clinical registration platforms (Cochrane Library, Chinese Cochrane Centre's Clinical Trial RegistryPlatform).

Main outcome(s): PubMed, EMBASE, the Cochrane Library, Chinese National Knowledge Infrastructure, Chinese VIP Information, Wanfang Database, and Chinese Biomedical Literature Database were searched by 2 reviewers from the inception until December 2021.The original study that randomised control trials of fire needle for RAS will be selected and is not limited by country or language. In addition, researches in progress, the reference lists and the citation lists of identified publications will be retrieved similarly. Study selection, data extraction, and assessment of the quality will be performed independently by 2 reviewers who have been trained prior to data extraction. A meta-analysis will be conduct if the 
quantity and quality of the original studies included are satisfactory; otherwise, a descriptive analysis will be conducted. Review Manager V5.4: (The Nordic Cochrane Centre, The Cochrane Collaboration, Copenhagen, Denmark) software will be using for data synthesis and assessment the risk of bias according by Cochrane Handbook. Results: This study will provide a comprehensive review of current evidence for the treatment of fire needle on RAS. Conclusion: The conclusion of this study will provide a judging basis that whether the treatment of RAS with fire needle is effective.

Data management: Study selection.All reviewers undergo rigorous training prior to selecting the study. Preliminary screening of the study will be conducted by 2 reviewers independently. After searching, the duplicated studies will be removal initially from the retrieved studies by Endnote (X9). And then, 2 independent reviewers will screened titles, abstracts, and keywords of all retrieved studies for candidates according to the inclusion and exclusion criteria, we will obtain the full text of all possibly relevant studies. Excluded studies will be recorded with explanations. If it is uncertain whether to adopt because of the lack of information, Jun Chen will try to contact authors of the original reports to obtain the information of lost. During the procedure, disagreements will be resolved by discussion or consensus with the third reviewer.Study selection will be performed in accordance with the Preferred Reporting Items for Systematic Reviews and Meta-analyses flowchart (Fig. 1). Data extraction and management. All candidate articles were evaluated and extracted by two independent authors. If disagreement occurred, two authors discussed and arrived at consensus with a third author.Information from each article will be recorded based on the following table in an Excel document; which includes first author, year of publication, country of publication, study design, total number of cases and gender, follow-ups, treatment strategy, control strategy, etc (Table 1).
Quality assessment / Risk of bias analysis: Assessment of risk of bias. To systematically evaluate the quality of each of the studies that final included. Two reviewers will assess the risk of bias for each included study according to the Cochrane handbook. It will eventually be rated on 3 levels ("high risk of bias", "medium risk of bias"and"low risk of bias"). The specific evaluation items include the following 7 aspects: generation of random sequence, allocation concealment, blindness of participants, and personnel, blindness of outcome assessment, incomplete outcome data, selective reporting and other bias.

Strategy of data synthesis: Data extraction and management. All candidate articles were evaluated and extracted by two independent authors. If disagreement occurred, two authors discussed and arrived at consensus with a third author.Information from each article will be recorded based on the following table in an Excel document; which includes first author, year of publication, country of publication, study design, total number of cases and gender, follow-ups, treatment strategy, control strategy, etc (Table 1).

Subgroup analysis: Data synthesis and subgroup analysis. All analysis will be done through RevMan 5.4. According to heterogeneity assessment, mean difference or relative risk were calculated using fixed or random effects models. In addition, if the I2obtained after data consolidation is greater than $50 \%$ and the Pvalue is less than .1, sensitivity or subgroup analysis will be performed to exclude the source of heterogeneity. If the included original research data is insufficient for quantitative analysis, the review will only represent, and summarize the evidence.

Sensitivity analysis: Sensitivity analysis. If the results show significant heterogeneity and the number of included studies is sufficient, sensitivity analysis will be performed to identify the quality and robustness of the meta-analysis result, which includes assessing the impact of sample size, methodological elements and 
the characteristic of research, and missing data.

Language: Language will be limited on English and Chinese.

Country(ies) involved: China.

Keywords: acupuncture, fire needle, recurrent aphthous stomatitis, protocol, systematic review.

Contributions of each author:

Author 1 - Jun Chen.

Email: juston521@163.com

Author 2 - Xingxin Wang.

Email: 1737788691@qq.com

Author 3 - Kai Wang.

Email: abc156639266@163.com

Author 4 - Zhihao Liu.

Email: nljhyshsdd@163.com

Author 5 - Xiaoya Lu.

Email: nldryqbhc@163.com

Author 6 - Ming Wang.

Email: wxx1737788691@163.com

Author 7 - Soudan Sun.

Email: fyhdiuscbhsch@163.com 